\title{
Functional Relationships between Agonist Binding Sites and Coupling Regions of Homomeric Cys-Loop Receptors
}

\author{
Natalia Andersen, ${ }^{1}$ Jeremías Corradi, ${ }^{1}$ Mariana Bartos, ${ }^{1}$ Steven M. Sine, ${ }^{2}$ and Cecilia Bouzat ${ }^{1}$ \\ ${ }^{1}$ Instituto de Investigaciones Bioquímicas, Universidad Nacional del Sur-Consejo Nacional de Investigaciones Científicas y Técnicas, Bahía Blanca 8000, \\ Argentina, and ${ }^{2}$ Receptor Biology Laboratory, Departments of Physiology and Biomedical Engineering and Neurology, Mayo Clinic College of Medicine, \\ Rochester, Minnesota 55905
}

Each subunit in a homopentameric Cys-loop receptor contains a specialized coupling region positioned between the agonist binding domain and the ion conductive channel. To determine the contribution of each coupling region to the stability of the open channel, we constructed a receptor subunit $\left(\alpha 7-5-\mathrm{HT}_{3 \mathrm{~A}}\right)$ with both a disabled coupling region and a reporter mutation that alters unitary conductance, and coexpressed normal and mutant subunits. The resulting receptors show single-channel current amplitudes that are quantized according to the number of reporter mutations per receptor, allowing correlation of the number of intact coupling regions with mean open time. We find that each coupling region contributes an equal increment to the stability of the open channel. However, by altering the numbers and locations of active coupling regions and binding sites, we find that a coupling region in a subunit flanked by inactive binding sites can still stabilize the open channel. We also determine minimal requirements for channel opening regardless of stability and find that channel opening can occur in a receptor with one active coupling region flanked by functional binding sites or with one active binding site flanked by functional coupling regions. The overall findings show that, whereas the agonist binding sites contribute interdependently and asymmetrically to open-channel stability, the coupling regions contribute independently and symmetrically.

\section{Introduction}

Receptors of the Cys-loop superfamily mediate rapid synaptic transmission throughout the nervous system and include receptors activated by the neurotransmitters ACh, GABA, glycine, and serotonin (for review, see Sine and Engel, 2006; Bartos et al., 2009; Thompson et al., 2010). Each member of this receptor family comprises a ring of five homologous subunits that together form multiple extracellular agonist binding sites and a single, spatially distant ion-conductive channel (Unwin, 2005). Cys-loop receptors function as chemical-to-electrical transducers through linkage of the binding sites to the channel via intervening coupling regions (Kash et al., 2003; Bouzat et al., 2004, 2008; Chakrapani et al., 2004; Grutter et al., 2005; Lee and Sine, 2005; Mercado and Czajkowski, 2006). The structural relationship between the binding sites and the coupling regions is novel, with each binding site formed at interfaces between pairs of subunits, but with each coupling region confined within each subunit. A fundamental question thus emerges of how the organization of binding sites and coupling regions optimizes opening of the channel.

\footnotetext{
Received Nov. 10, 2010; revised Dec. 23, 2010; accepted Jan. 9, 2011.

This work was supported by National Institutes of Health Grant NS053521 (S.M.S., C.B.) and grants from Universidad Nacional del Sur and Agencia Nacional de Promoción Científica y Tecnológica Argentina (C.B.). We thank Chris Free for technical contributions.

Correspondence should be addressed to either of the following: Dr. Steven M. Sine, Mayo Clinic College of Medicine, Rochester, MN 55905, E-mail: sine@mayo.edu; or Dr. Cecilia Bouzat, Instituto de Investigaciones Bioquímicas, Universidad Nacional del Sur-Consejo Nacional de Investigaciones Científicas y Técnicas, Bahía Blanca 8000, Argentina, E-mail: inbouzat@criba.edu.ar.

DOI:10.1523/JNEUROSCI.5940-10.2011

Copyright $\odot 2011$ the authors $\quad 0270-6474 / 11 / 313662-08 \$ 15.00 / 0$
}

Our recent studies addressed the question of the organization of the agonist binding sites using a model homomeric Cys-loop receptor composed of the ligand binding domain of the neuronal nicotinic $\alpha 7$ receptor and the channel and cytoplasmic domains of the $5-\mathrm{HT}_{3 \mathrm{~A}}$ receptor (Rayes et al., 2009). Because the agonist binding sites of Cys-loop receptors are formed at interfaces between subunits (for review, see Sine, 2002), and homomeric receptors contain five identical subunits, our model receptor contains five identical binding sites. By coexpressing normal subunits with subunits mutated to form a disabled binding site, we varied the number and positioning of the binding sites within the pentamer. To correlate the number of disabled binding sites with the stability of the open channel, we developed an electrical fingerprinting strategy in which a reporter mutation that altered the single-channel current amplitude, but not open-channel stability, was installed in the subunit with a binding site disabling mutation. Thus, patch-clamp recordings simultaneously registered the open duration and number of intact agonist binding sites for each receptor that elicited a channel opening event. The findings showed that, of the five binding sites per receptor, three sites at nonconsecutive subunit interfaces were the minimal requirement for maximal channel open duration (Rayes et al., 2009). Here, we use the electrical fingerprinting strategy to determine the relationship between the number of coupling regions per pentameric receptor and channel open duration. Furthermore, we use the same strategy to examine the relationship between the number and locations of binding sites and coupling regions.

\section{Materials and Methods}

Site-directed mutagenesis and expression of $\alpha 7-5-H T_{3 A}$ receptors. The chimeric receptor composed of the extracellular domain of human $\alpha 7$ and 
the transmembrane domain of mouse $5-\mathrm{HT}_{3 \mathrm{~A}}$ receptor was used as a model homomeric Cys-loop receptor (Eiselé et al., 1993; Rayes et al., 2005). The high-conductance form (HC) of the $\alpha 7-5-\mathrm{HT}_{3 \mathrm{~A}}$ chimeric receptor was constructed as described previously (Rayes et al., 2005; Bouzat et al., 2008). In brief, three arginine residues at the intracellular M3-M4 loop responsible for the low conductance of the $5-\mathrm{HT}_{3 \mathrm{~A}}$ receptor were mutated to glutamine $(\mathrm{Q})$, aspartic acid $(\mathrm{D})$, and alanine $(\mathrm{A})$ (Kelley et al., 2003). Mutant subunits were constructed using the QuikChange Site-Directed Mutagenesis kit (Stratagene) and confirmed by sequencing the entire cDNA insert. BOSC cells were transfected with subunit cDNAs using calcium phosphate precipitation. For a $35 \mathrm{~mm}$ dish of cells, the total amount of cDNA was $5 \mu \mathrm{g}$, and the ratios of control and mutant subunit cDNAs are described in the text for each experiment. Cells were used for single-channel or macroscopic current measurements 1 or $2 \mathrm{~d}$ after transfection (Bouzat et al., 1994, 2002).

The apparent dissociation constant for $\mathrm{ACh}, K_{\mathrm{d}}$, was determined by competition of specified concentrations of ACh against the initial rate of ${ }^{125} \mathrm{I}$ - $\alpha$-bungarotoxin $(\alpha$-BTX) binding as previously described (Sine et al., 1995; Bouzat et al., 2008).

Single-channel patch-clamp recordings. Single-channel recordings were obtained in the cell-attached patch configuration (Hamill et al., 1981). The bath and pipette solutions contained $142 \mathrm{~mm} \mathrm{KCl}, 5.4 \mathrm{~mm} \mathrm{NaCl}, 0.2$ $\mathrm{mM} \mathrm{CaCl}_{2}$, and $10 \mathrm{~mm} \mathrm{HEPES}$, pH 7.4. Solutions free of magnesium and with low-calcium were used to minimize channel block by divalent cations (Rayes et al., 2005). Single-channel currents were recorded and lowpass filtered at a cutoff frequency of $10 \mathrm{kHz}$ using an Axopatch 200B patch-clamp amplifier (Molecular Devices), digitized at $5 \mu$ s intervals, and detected by the half-amplitude threshold criterion using the program TAC (Bruxton Corporation) (Bouzat et al., 2004). Open-time histograms were fitted by the sum of exponential functions by maximum likelihood using the program TACFit (Bruxton Corporation). Because no correction for missed events was applied, open durations should be considered apparent open times.

To define amplitude classes, an analysis of the whole recording was performed by tracking events regardless of current amplitude. Amplitude histograms were then constructed and the different amplitude classes were distinguished. At least 10 different recordings from cells transfected with different ratios of HC and low-conductance (LC) subunits were analyzed to define the mean \pm SD of each amplitude class (Rayes et al., 2009).

To determine the mean open and burst durations of each amplitude class, the analysis was performed in two different ways. In the first, all opening events were detected without any restriction of amplitude as described above. Open time histograms were then constructed for a given amplitude class by selecting only openings with amplitudes of $\pm 0.6 \mathrm{pA}$ of that of the mean of the amplitude class. This procedure involved the detection of all events in the whole recording in only one step. In the second way, only channel openings whose amplitudes were between $\pm 0.6 \mathrm{pA}$ of that of the mean amplitude class under study were accepted during the detection. This was performed by setting the detection bar to a fixed amplitude corresponding to the mean of the class of interest, then detecting channel events by the half-amplitude threshold criterion, and finally manually discarding events if the amplitude differed by $>0.6 \mathrm{pA}$ of that of the mean amplitude class under study. The corresponding duration histogram was then constructed from the accepted events. Thus, this procedure involves analyzing the recording all the way through for each amplitude class. No significant differences in the mean open and burst durations were observed between the two types of analyses.

To identify bursts and quantify their durations, a critical closed time $\left(\tau_{\text {crit }}\right)$ was defined as the point of intersection between the second briefest and the succeeding components, and openings separated by closings briefer than this time constitute a burst (Rayes et al., 2005). Typical $\tau_{\text {crit }}$ values ranged from 1 to $2 \mathrm{~ms}$. Burst duration was taken from the duration of the slowest open component.

For single-channel recordings, we routinely used a concentration of 1 mm because ACh blocks the open channel at higher concentrations. Nevertheless, we tested $2 \mathrm{~mm}$ ACh in mutants with fewer than five active binding sites and did not find any difference between recordings at 1 and $2 \mathrm{~mm}$. We showed previously that this chimeric receptor exhibits long openings and bursts at ACh concentrations as low as $30 \mu \mathrm{M}$. The durations of the brief, intermediate, and long open or burst components do not change with ACh concentration and the areas of the components remain constant at concentrations of $>30 \mu \mathrm{M}$ ACh (Rayes et al., 2005).

Macroscopic current recordings. For whole-cell recordings, a series of $1.5 \mathrm{~s}$ pulses of extracellular solution containing ACh were applied (Liu and Dilger, 1991; Rayes et al., 2005). The pipette solution contained 134 mM KCl, 5 mm EGTA, $1 \mathrm{~mm} \mathrm{MgCl}_{2}$, and $10 \mathrm{~mm}$ HEPES, pH 7.3. The extracellular solution (ECS) contained $150 \mathrm{~mm} \mathrm{NaCl}, 5.6 \mathrm{~mm} \mathrm{KCl}, 0.5$ $\mathrm{mm} \mathrm{CaCl}_{2}$, and $10 \mathrm{~mm}$ HEPES, $\mathrm{pH}$ 7.3. The perfusion system consists of solution reservoirs, manual switching valves, a four channel $\mathrm{VC}^{3}$ valve controller (ALA Scientific Instruments), a solenoid-driven pinch valve, and two tubes (inner diameter, $0.3 \mathrm{~mm}$ ) orientated at $90^{\circ}$ inserted into the culture dish (Liu and Dilger, 1991). One tube contained the ECS without agonist and the other contained ECS with ACh. Macroscopic currents were recorded at a holding potential of $-50 \mathrm{mV}$, filtered at 5 $\mathrm{kHz}$, and digitized at $20 \mathrm{kHz}$. Data analysis was performed using IgorPro software (Wavemetrics). Individual records were aligned at the point at which the current reached $50 \%$ of maximum, and the resulting records were averaged. The solution exchange time was estimated by positioning an open pipette close to the cell and switching from normal bath solution to a diluted (1:1 with water) bath solution. For whole-cell recordings, typical exchange times ranged from 1 to $2 \mathrm{~ms}$ (Bouzat et al., 2008). The $\mathrm{EC}_{50}$ value for ACh activation of this chimera is $\sim 200 \mu \mathrm{M}$ (Rayes et al., 2005). Macroscopic responses were elicited by $1 \mathrm{~mm}$ ACh at which saturation is achieved. A concentration of $10 \mathrm{~mm}$ was used to confirm lack of macroscopic responses for nonfunctional mutants.

\section{Results}

The experiments herein aim to determine how the number of coupling regions per homopentameric Cys-loop receptor affects the stability of the open state of the channel. Thus, the first step was to identify a mutation within the coupling region that prevents channel opening yet retains the capacity for agonist binding. Additionally, the ideal mutant subunit would express well alone, assemble with normal subunits, and not affect singlechannel conductance. As in our previous study of binding site stoichiometry and positioning (Rayes et al., 2009), our model Cys-loop receptor is the $\alpha 7-5-\mathrm{HT}_{3 \mathrm{~A}}$ chimera (Eiselé et al., 1993). The following sections describe modifications of this chimera that produce receptors satisfying these requirements.

The coupling region comprises four structures from the ligand binding domain ( $\beta 1-\beta 2, \beta 8-\beta 9, \beta 10$, and Cys-loop) and two structures from the channel domain (pre-M1 and M2-M3). Numerous studies demonstrate that these regions, individually and in combination, contribute to transduction of agonist binding to channel gating (Kash et al., 2003; Bouzat et al., 2004; Xiu et al., 2005; Lee et al., 2009). Our previous studies using $\alpha 7-5-\mathrm{HT}_{3 \mathrm{~A}}$ chimeras showed that the pre-M1 region interacts with the M2-M3 region and that a mismatch between these regions prevents transduction (Bouzat et al., 2008). In particular, we found that substitutions in the pre-M1 region, changing the $5-\mathrm{HT}_{3 \mathrm{~A}}$ sequence PLFYAVS to the $\alpha 7$ sequence TLYYGLN, prevent agonistevoked currents (Fig. 1). Furthermore, when expressed in BOSC 23 cells, this mutant subunit yielded abundant quantities of ${ }^{125} \mathrm{I}-\alpha$-BTX binding sites on the cell surface $\left(84 \pm 7 \%\right.$ relative to the $\alpha 7-5-\mathrm{HT}_{3 \mathrm{~A}}$ chimera), and exhibited only a slight increase of the apparent dissociation constant for ACh binding $\left(K_{\mathrm{d} \text { control }}=56 \pm 4.2 \mu \mathrm{M}\right.$; $K_{\text {d TLYYGLN }}=86 \pm 8.7 \mu \mathrm{M}$ ) (Bouzat et al., 2008).

To ensure that the TLYYGLN mutant completely prevents channel opening, we recorded both macroscopic and singlechannel currents from BOSC 23 cells transfected with either the control $\alpha 7-5-\mathrm{HT}_{3 \mathrm{~A}}$ chimera or the TLYYGLN mutant. To increase single current amplitude in both the control and mutant constructs, three arginine residues in the cytoplasmic domain 


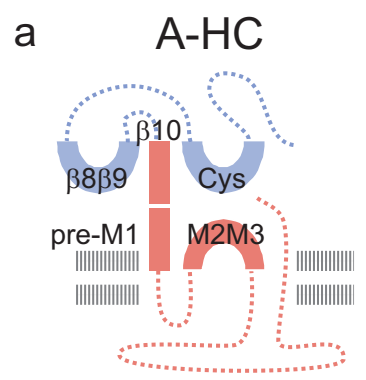

Active

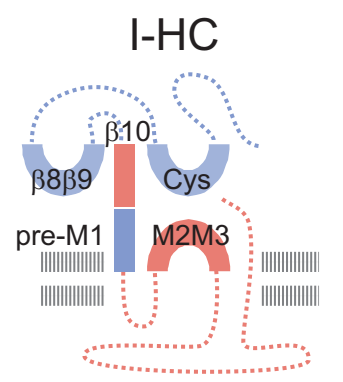

Inactive

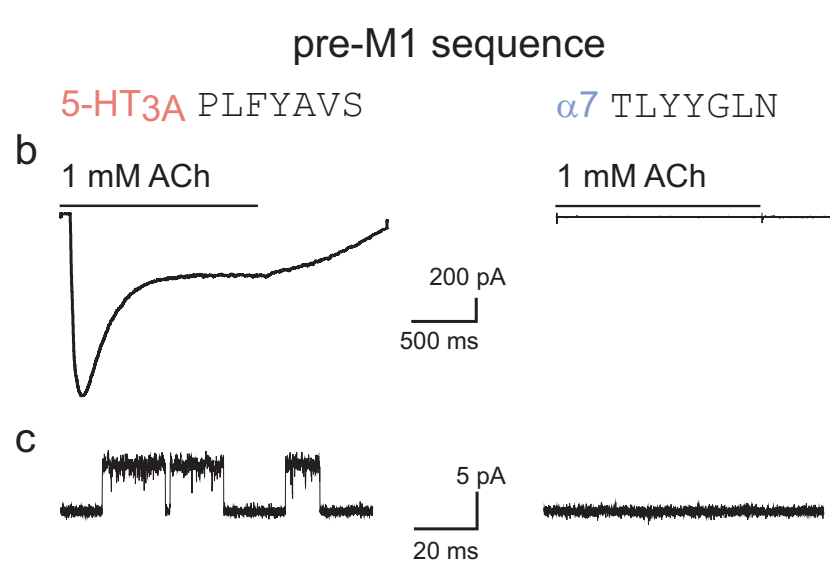

Figure 1. Mutations at the coupling region decouple agonist binding from channel gating. $\boldsymbol{a}$, Schematic diagrams of receptor subunits with the loops located at the coupling region, with each color representing a different segment of the linear sequence; $\alpha 7$ is shown in blue and $5-\mathrm{HT}_{3 \mathrm{~A}}$ in red. The original $\alpha 7-5-\mathrm{HT}_{3 \mathrm{~A}}$ chimera contains an active coupling region (A-HC). Replacement of the pre-M1 region from $5-\mathrm{HT}_{3 \mathrm{~A}}$ to $\alpha 7$ sequence (I-HC) completely abolishes channel function. $\boldsymbol{b}$, Macroscopic current responses to $1500 \mathrm{~ms}$ pulses of $1 \mathrm{~mm}$ ACh obtained in the whole-cell configuration. Holding potential, $-50 \mathrm{mV}$.c, Single-channel recordings in the presence of $1 \mathrm{~mm}$ ACh. Membrane potential, $-70 \mathrm{mV}$; filter, $10 \mathrm{kHz}$. In all single-channel current traces, channel openings are shown as upward deflections. No channel activity is detected for the M1-mutant chimera (I-HC).

were mutated to glutamine, aspartic acid, and alanine (Kelley et al., 2003); these mutations increase current amplitude from undetectable to $\sim 10 \mathrm{pA}$ at a membrane potential of $-120 \mathrm{mV}$, but open-channel lifetime is not affected (Bouzat et al., 2008). To distinguish transfected from nontransfected cells, a cDNA encoding green fluorescent protein was included in all transfections. Application of $1 \mathrm{~mm}$ ACh to cells expressing the control $\alpha 7-5-\mathrm{HT}_{3 \mathrm{~A}}$ chimera elicits rapidly activating and slowly desensitizing macroscopic currents (Fig. 1). However, when applied to cells expressing the mutant chimera, ACh does not elicit macroscopic currents (30 whole-cell recordings from cells exhibiting green fluorescence).

Because even a low frequency of channel opening could produce an aberrant signal in electrical fingerprinting, we looked for ACh-evoked single-channel currents. In the presence of $1 \mathrm{~mm}$ ACh, the control $\alpha 7-5-\mathrm{HT}_{3 \mathrm{~A}}$ chimera produces robust singlechannel activity in which openings appear either alone or in groups of several openings in quick succession. By contrast, the TLYYGLN mutant shows no single-channel activity (50 patches from cells exhibiting green fluorescence) (Fig. 1). Thus, hereafter we refer to the TLYYGLN mutant chimera as I-HC, I for inactive coupling region and $\mathrm{HC}$ for high conductance. Analogously, the control $\alpha 7-5-\mathrm{HT}_{3 \mathrm{~A}}$ chimera is named A-HC, A for active coupling region and $\mathrm{HC}$ for high conductance.

The electrical fingerprinting approach is illustrated in Figure $2 a$. The left panel shows recordings in the presence of $1 \mathrm{~mm} \mathrm{ACh}$ from cells transfected with A-HC and reveals a large and uniform single-channel current amplitude. The right panel shows recordings from cells transfected with A-HC and A-LC subunits in which LC stands for low conductance because of retention of the three native arginine residues in the cytoplasmic domain. Combination of the two different subunits, by contrast, yields singlechannel amplitudes with five equally spaced conductance levels, ranging from $\sim 1.3$ to $10 \mathrm{pA}$ (Fig. 2). Because the receptor is pentameric, the five single-channel current amplitudes arise from different numbers of HC subunits per receptor. Thus, amplitude classes of $10,8,5.8,3.8$, and $1.3 \mathrm{pA}$ correspond to receptors containing 5, 4, 3, 2, and $1 \mathrm{HC}$ subunit, respectively (Rayes et al., 2009).

Returning to the question of coupling region stoichiometry, we sought to further insure that the I-HC mutant does not affect single-channel current amplitude. Because the I-HC subunit expressed alone does not produce single-channel currents, we coexpressed it with A-HC in a 1:1 ratio. Recordings in the presence of $1 \mathrm{~mm}$ ACh revealed frequent single-channel openings with a single Gaussian distributed current amplitude of $\sim 10 \mathrm{pA}$ (Fig. $2 b$, left panel). Assuming the I-HC and A-HC subunits coassemble, the absence of openings with different current amplitudes shows that the TLYYGLN mutation does not affect single-channel conductance.

To test whether the subunit with the TLYYGLN mutation coassembles with normal subunits, we coexpressed A-HC and I-LC subunits (Fig. $2 b$, right panel). Recordings from cells transfected with this subunit combination revealed frequent singlechannel openings. As shown for the combination A-HC plus A-LC (Fig. 2a), the single-channel current amplitudes spanned a range from $\sim 1.3$ to $10 \mathrm{pA}$ (Fig. $2 b$ ). After measuring the current amplitude of each channel opening event, an amplitude histogram revealed a distribution with four equally spaced peaks well fitted by the sum of four Gaussian functions (Fig. 2b). As in Figure $2 a$, the peaks arise from receptors with different numbers of A-HC subunits; a fifth peak corresponding to one A-HC subunit per receptor is expected, but these events, although visible, were not detected using the threshold crossing method because of the low signal-to-noise ratio inherent to small amplitude events. Thus, the I-LC subunit coassembles with A-HC subunits, and the combination produces single-channel current amplitudes that are quantized according to the number of A-HC subunits per receptor.

Recordings from receptors produced by A-HC and I-LC subunits provide initial insights into how coupling region stoichiometry impacts stability of the open channel. In additional studies, the subunit ratio was varied from 1:1 to 1:4 to enhance the probability of obtaining openings with a desired amplitude class, which can be estimated by the binomial distribution. Openings with amplitude of $10 \mathrm{pA}$ correspond to receptors with five highconductance subunits and, therefore, five intact coupling regions (Fig. 3, 5 A-HC), and yield open time histograms described by the sum of three exponential components with time constants $\tau_{1}=$ $130 \pm 17 \mu \mathrm{s}, \tau_{2}=1.1 \pm 0.3 \mathrm{~ms}$, and $\tau_{3}=8.8 \pm 2.3 \mathrm{~ms}$ (Fig. 3 , Table 1). This distribution of open dwell times is essentially the same as that obtained from cells transfected with A-HC alone (Rayes et al., 2005; Bouzat et al., 2008). The brief and intermediate duration openings arise mainly from isolated single openings, whereas the long duration openings arise from bursts of several openings in quick succession (Rayes et al., 2005).

To determine the distribution of burst durations, we defined a burst as a series of closely spaced openings preceded and followed by a closed interval greater than a specified duration; typically, this 


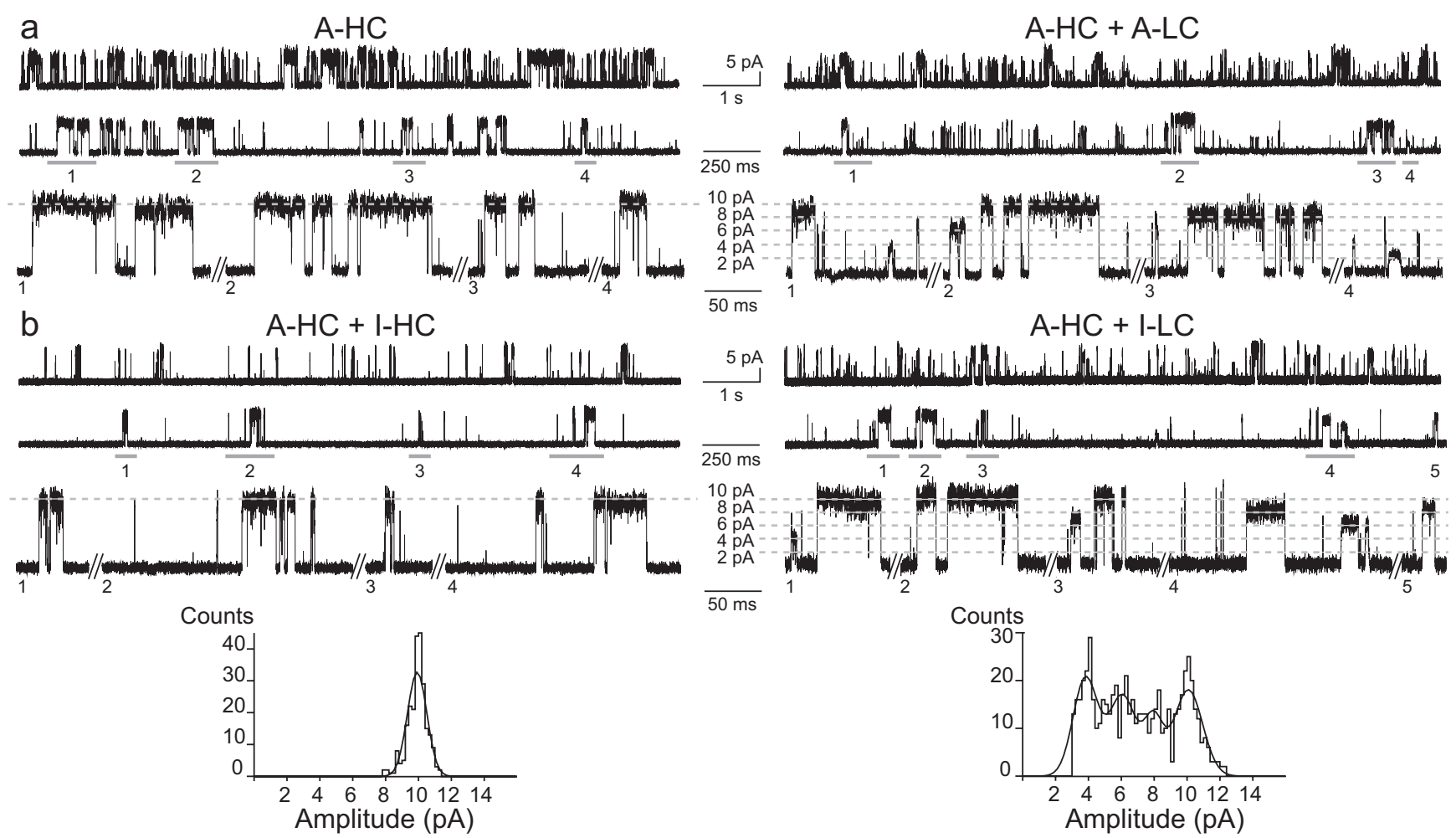

Figure 2. Coexpression of high-and low-conductance forms of the subunits. Single-channel recordings were obtained in the presence of $1 \mathrm{~mm} \mathrm{ACh}$ at a membrane potential of $-120 \mathrm{mV}$; filter, $9 \mathrm{kHz}$. , Cells were cotransfected with A-HC (left) or A-HC and A-LC (right panel) in a 1:1 subunit ratio. Channel traces are shown at three different timescales. Segments from the $250 \mathrm{~ms}$ traces (marked with numbers) are shown below at higher time resolution ( $50 \mathrm{~ms}$ scale). Currents through the $\mathrm{HC}$ form of $\alpha 7-5-\mathrm{HT} 3 \mathrm{~A}(\mathrm{~A}-\mathrm{HC})$ show a single class of current amplitude, whereas discrete amplitude classes are observed from cells transfected with both high- and low-conductance forms (A-HC plus A-LC). $\boldsymbol{b}$, Cells were cotransfected with A-HC and I-HC (left) or A-HC and I-LC (right panel) in a 1:1 subunit ratio. Channel traces are shown at three different timescales. Segments from the $250 \mathrm{~ms}$ traces (marked with numbers) are shown below at higher time resolution ( $50 \mathrm{~ms})$. Note multiple discrete amplitude classes are detected only in cells transfected with A-HC plus I-LC subunits. Amplitude histograms from whole recordings obtained from cells transfected with A-HC plus I-HC (left) or A-HC plus I-LC (right) are shown at the bottom. Note that, for A-HC plus $\mathrm{I}-\mathrm{LC}$, the amplitude classes of 10,8,5.8, and $3.8 \mathrm{pA}$ are clearly distinguished. Channels of smaller amplitudes were not analyzed.

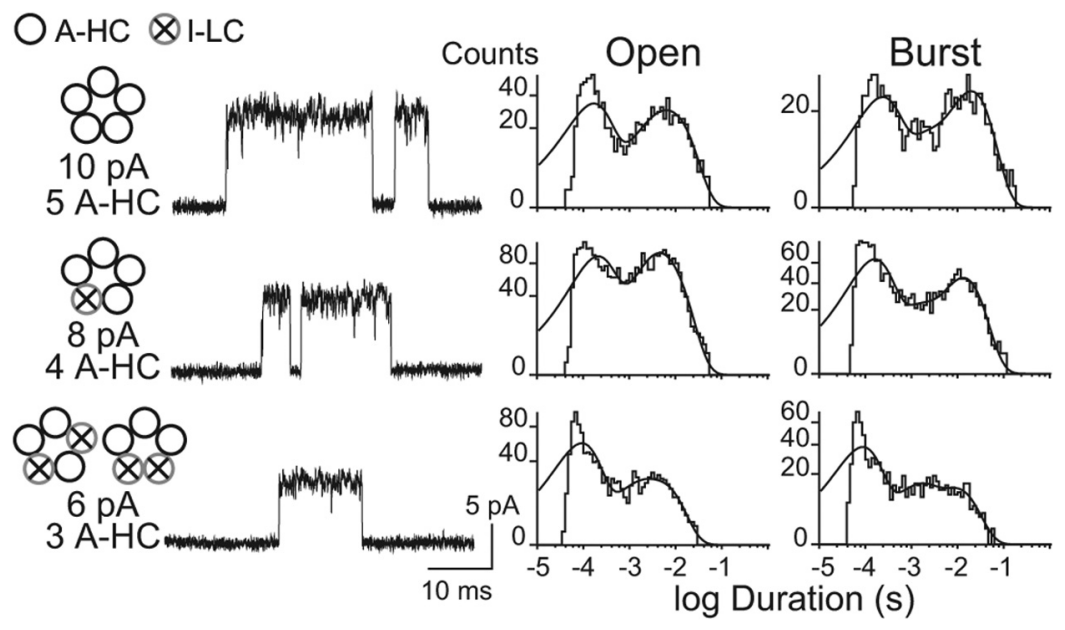

Figure 3. Activation of receptors with five, four, or three active coupling regions. Cells were cotransfected with A-HC and I-LC subunit CDNAs using different subunit ratios. Channel recordings were performed in the presence of $1 \mathrm{~mm} A C h$ at a membrane potential of $-120 \mathrm{mV}$; filter, $9 \mathrm{kHz}$. Arrangements of subunits giving rise to amplitude classes of $10 \mathrm{pA}$ ( $5 \mathrm{HC}$ subunits and 5 active coupling regions), $8 \mathrm{pA}$ (4 active coupling regions), and $6 \mathrm{pA}$ (3 active coupling regions) are shown in the left. In the schemes, the black cross indicates inactive coupling region, and high- and low-conductance subunits are shown as black and gray circles, respectively. Open and burst duration histograms were constructed after selecting events corresponding to each amplitude class, plotted on a logarithmic timescale and fitted by the sum of three exponentials.

duration was 1-2 ms (see Materials and Methods) (Rayes et al., 2005). The resulting burst duration histogram is described by the sum of three exponential components, two of which have the same time constants as in the open duration histogram, whereas the mean duration of the third component increases approximately twofold to a $\tau_{\mathrm{B}}$ of $17.8 \mathrm{~ms}$ (Table 1, line 1). Because the majority of current through $\alpha 7-5-\mathrm{HT}_{3 \mathrm{~A}}$ receptors flows during bursts of openings, we considered these to be of greatest functional significance. Thus, we used mean open time and mean burst duration as indicators of open-channel stability.

Openings with amplitude of $8 \mathrm{pA}$ are readily detected using an A-HC to I-LC subunit ratio of $1: 1$, and correspond to receptors with four intact coupling regions (Fig. 3, 4 A-HC). Open and burst duration histograms are again fitted by the sum of three exponential components. The open and burst components with longest mean duration show $\tau_{\mathrm{O}}=7 \mathrm{~ms}$ and $\tau_{\mathrm{B}}=14 \mathrm{~ms}$, significantly briefer than those from receptors with five intact coupling regions $(p<0.05)$ (Table 1, line 2).

Openings with amplitude of $6 \mathrm{pA}$ are also readily detected and correspond to receptors with three high-conductance subunits and, therefore, three intact coupling regions (3 A-HC) (Fig. 3). The open and burst components with longest mean duration show $\tau_{\mathrm{O}}=5.5 \mathrm{~ms}$ and $\tau_{\mathrm{B}}=10.7 \mathrm{~ms}$, which are briefer than those from receptors with four or five intact coupling regions (Table 1, line 3 ). However, the mean 
Table 1. Open and burst durations for receptors with different numbers of active coupling regions

\begin{tabular}{|c|c|c|c|c|c|c|c|}
\hline No. of active regions & \multicolumn{2}{|c|}{ Subunit (number in the arrangement) } & Receptor & $\mathrm{pA}$ & $\tau_{0}$ (ms) & $\tau_{\mathrm{B}}(\mathrm{ms})$ & $n$ \\
\hline 5 & $\mathrm{~A}-\mathrm{HC}(5)$ & I-LC(0) & & $9.9 \pm 0.6$ & $8.8 \pm 2.3$ & $17.8 \pm 5.0$ & 15 \\
\hline 4 & $\mathrm{~A}-\mathrm{HC}(4)$ & I-LC(1) & & $7.9 \pm 0.3$ & $7.0 \pm 1.2$ & $14.0 \pm 3.3$ & 14 \\
\hline 3 & $\mathrm{~A}-\mathrm{HC}(3)$ & I-LC(2) & & $5.8 \pm 0.3$ & $5.5 \pm 2.8$ & $10.7 \pm 5.2$ & 13 \\
\hline 2 & $A-L C(2)$ & $\mathrm{I}-\mathrm{HC}(3)$ & & $6.1 \pm 0.3$ & $4.9 \pm 1.1$ & $7.6 \pm 1.5$ & 10 \\
\hline 1 & $A-L C(1)$ & $\mathrm{I}-\mathrm{HC}(4)$ & & $8.1 \pm 0.1$ & $3.0 \pm 0.8$ & $7.4 \pm 3.0$ & 6 \\
\hline
\end{tabular}

Single-channel currents were recorded from cells transfected either with A-HC plus I-LC or with A-LC plus I-HC subunit CDNAs. Possible subunit arrangements for each channel amplitude class are shown. In the schemes, the black cross indicates inactive coupling region, and high- and low-conductance forms are shown as black and gray circles, respectively. $\tau_{0}$ and $\tau_{\mathrm{B}}$ correspond to the longest duration component of the open and burst duration histograms, respectively. Results are shown as mean \pm SD for each amplitude class for the indicated number of patches, $n$.

durations exhibited greater than usual variability from one recording to the next, as indicated by the greater SDs (Table 1). A possible explanation for this increased variability is that the two arrangements of subunits with inactive coupling regions, consecutive or alternating (Table 1), may contribute somewhat differently to the stability of the open channel, analogous to the different extents of open-channel stabilization conferred by consecutive versus alternating positioning of two or three agonist binding sites (Rayes et al., 2009). Unlike the agonist binding sites, however, the coupling regions are confined within each subunit; thus, we could not discriminate between the two different arrangements.

Openings to the lower $\sim 3.8$ and $\sim 1.3$ pA current levels were not analyzed because measurements of open durations became less accurate because of a reduced signal-to-noise ratio. To cover the entire range of active coupling regions per receptor, we recorded from cells transfected with the reverse combination of subunits, A-LC and I-HC (Fig. 4). For this combination, openings of $10 \mathrm{pA}$ are not detected because they correspond to receptors with five inactive coupling regions. With the subunit ratio $1: 1$, the most frequent channel opening events correspond to the $6 \mathrm{pA}$ amplitude class corresponding to receptors with two active coupling regions (Fig. $4 a$ ). Mean open and burst durations are significantly briefer than those observed for receptors with three active coupling regions, showing $\tau_{\mathrm{O}}=4.9 \mathrm{~ms}$ and $\tau_{\mathrm{B}}=7.6$ ms (Fig. 4a; Table 1, line 4). There are two possible arrangements of two subunits with active coupling regions, but the relatively small SDs of the mean open and burst durations suggest they do not differ significantly in their open-channel stability.

To increase the probability of openings with $8 \mathrm{pA}$ amplitude, corresponding to receptors with one active coupling region, we

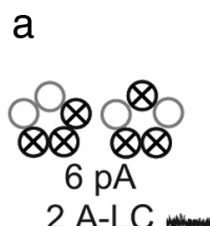

b

C (right).

\section{OA-LC QI-HC}
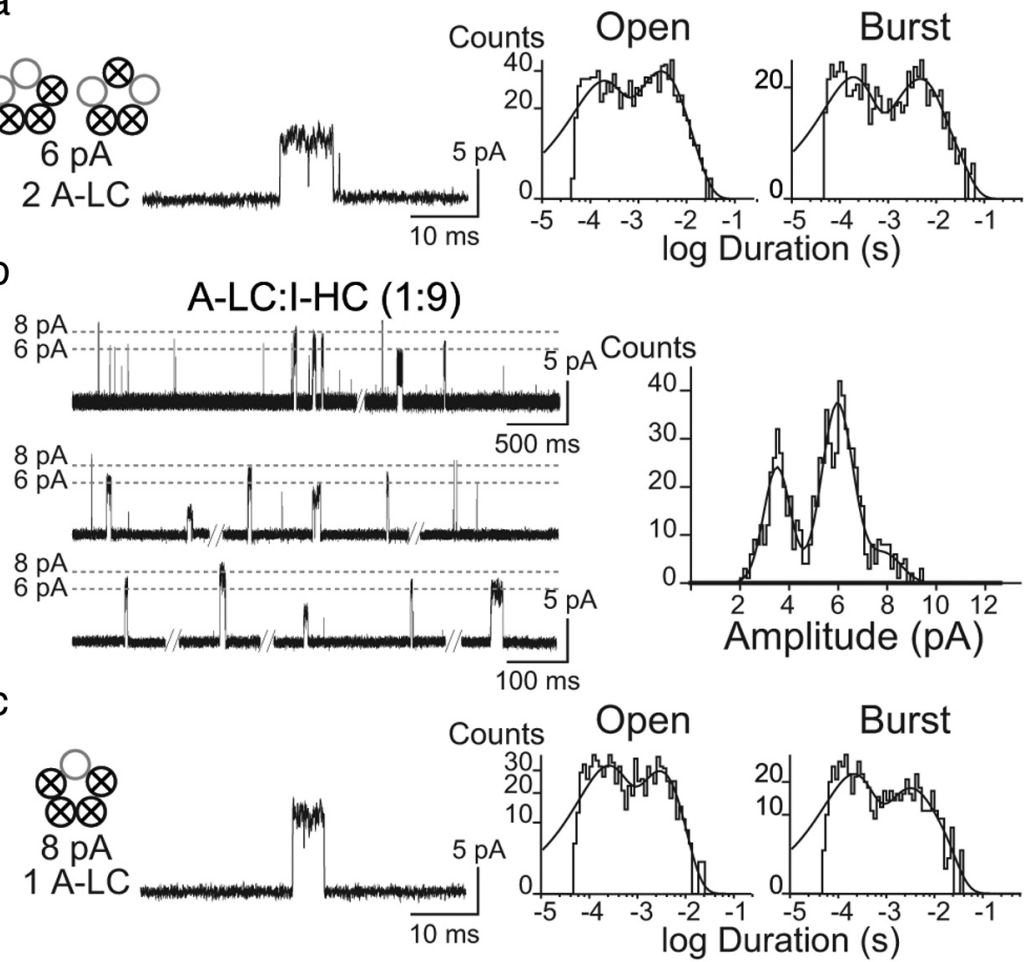

Figure 4. Activation of receptors with two or one active coupling regions. Cells were cotransfected with $\mathrm{A}-\mathrm{LC}$ and I-HC subunit CDNAs using different subunit ratios. Recordings were obtained in the presence of $1 \mathrm{~mm} \mathrm{ACh}$ at a membrane potential of $-120 \mathrm{mV}$; filter, $9 \mathrm{kHz}$. Open and burst duration histograms were constructed after selecting events corresponding to each amplitude class. $\boldsymbol{a}$, Arrangements of subunits in receptors giving rise to the $6 \mathrm{pA}$ amplitude class containing two active coupling regions (left). Schemes of subunits are as in Figure 3. Channel traces (middle) and open and burst duration histograms fitted by the sum of three exponentials (right) are shown. $\boldsymbol{b}$, Continuous traces of a single-channel recording from a cell transfected with A-LC/I-HC ratio of 1:9 (1 mM ACh; $-120 \mathrm{mV})$. The amplitude histogram for the corresponding entire recording is shown right. Note the low proportion of channels of the $8 \mathrm{pA}$ amplitude class. $c$, Arrangements of subunits for receptors giving rise to the amplitude class of $8 \mathrm{pA}$ that contains only one active coupling region (left), channel traces (middle), and corresponding open and burst duration histograms

increased the A-LC to I-HC ratio to 1:4 and 1:9. For these ratios, the binomial distribution predicts probabilities of 0.32 and 0.59 , respectively, for observing openings with $8 \mathrm{pA}$ amplitude. Nevertheless, only $\sim 20 \%$ of openings corresponded to the 8 pA class (Fig. $4 b$ ), suggesting receptors with only one active coupling region markedly decrease the probability of channel opening. For 


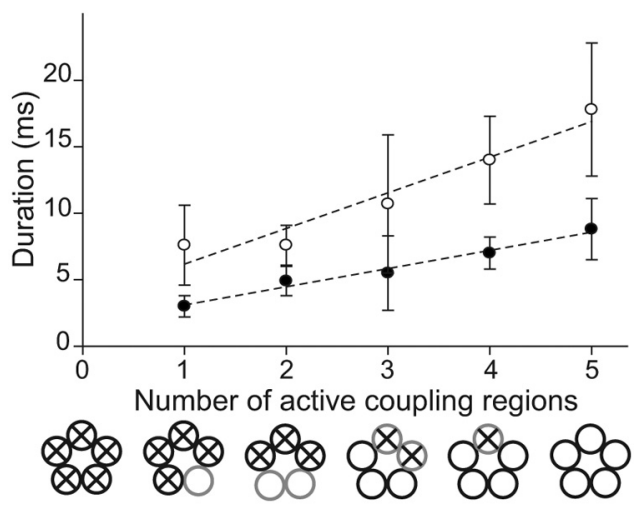

Figure 5. Channel lifetime $(\bigcirc)$ and burst duration $(\bigcirc)$ as a function of the number of active coupling regions. Data are from Table 1 and fitted by linear regression. Slopes of the lines are 1.4 $\mathrm{ms} /$ subunit $\left(r^{2}=0.98\right)$ and $2.7 \mathrm{~ms} /$ subunit $\left(r^{2}=0.93\right)$ for open time and burst duration, respectively.

the $8 \mathrm{pA}$ openings, mean open and burst durations were reduced further to $\tau_{\mathrm{O}}=3 \mathrm{~ms}$ and $\tau_{\mathrm{B}}=7.4 \mathrm{~ms}$ (Fig. $4 c$; Table 1, line 5).

A plot of mean open duration against the number of active coupling regions is linear and indicates each coupling region contributes $1.4 \mathrm{~ms}$ to open-channel lifetime (Fig. 5). Similarly, a plot of mean burst duration against the number of active coupling regions is approximately linear, with each coupling region contributing $2.7 \mathrm{~ms}$ to burst duration. The linear relationships further show that each coupling region contributes independently and symmetrically to channel open and burst durations.

The overall findings from this and our previous study show that both the agonist binding sites and the coupling region contribute to receptor function in an allosteric manner. Evidence for this is that changes in the number of either type of functional unit affect a global measure of receptor function, open-channel stability. However, each type of unit contributes differently. Decreasing the number of binding sites from five to three does not affect open-channel stability, as long one binding site is not adjacent to the other two. Yet decreasing the number of coupling regions from five to three significantly reduces openchannel stability. We therefore sought to examine the relationship between the number and positioning of the binding sites and coupling regions.

To this end, we combined mutations that disable the coupling region with mutations that disable the binding site (Rayes et al., 2009). Initially, we asked whether an active binding site requires an active coupling region in both subunits that contribute to the site. Thus, we coexpressed a range of subunit ratios of I-HC with either A-HC-Y190T that disables the principal face of the binding site or A-HC-W55T that disables the complementary face. The possible combinations of subunits within pentameric receptors are shown in Figure 6 for one of the binding site mutants. The combinations of subunits produce pentameric arrangements ranging from four functional binding sites and one active coupling region to one functional binding site and four active coupling regions. With a $1: 1$ ratio of subunits, the binomial distribution predicts a probability of 0.62 of obtaining receptors with two or three active binding sites. Ratios of 3:1 and 1:3 were also tested to cover a broad spectrum of possible arrangements. In all cases when both faces of a binding site are intact, only one or none of the constituent subunits contain an intact coupling region (Fig. 6).

Application of $10 \mathrm{~mm}$ ACh to cells transfected with any of the combinations of subunits failed to elicit macroscopic currents
Active coupling region $\bigotimes$ Inactive coupling region

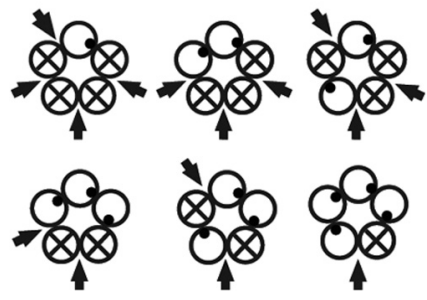

Figure 6. Possible subunit arrangements after cotransfection of $\mathrm{I}-\mathrm{HC}$ with either A-HCY190T or A-HC-W55T subunits. Inactive coupling region is shown by the black cross and the binding site mutation by the small filled circle. The arrows indicate functional binding sites.

Table 2. Open and burst durations for receptors with different numbers of active coupling regions and binding sites

\begin{tabular}{|c|c|c|c|c|c|c|c|c|}
\hline $\begin{array}{l}\text { No. of } \\
\text { active regions }\end{array}$ & $\begin{array}{l}\text { No. of } \\
\text { binding sites }\end{array}$ & $\begin{array}{l}\text { Subuni } \\
\text { in arrar }\end{array}$ & $\begin{array}{l}\text { it (number } \\
\text { ngement) }\end{array}$ & Receptor & $\mathrm{pA}$ & $\begin{array}{l}\tau_{0} \\
\text { (ms) }\end{array}$ & $\begin{array}{l}\tau_{\mathrm{B}} \\
\text { (ms) }\end{array}$ & $n$ \\
\hline 4 & 3 & $\begin{array}{l}\mathrm{A}-\mathrm{HC} \\
(4)\end{array}$ & $\begin{array}{l}\text { I-LC } \\
\text { Y190T } \\
\text { W55T } \\
(1)\end{array}$ & & 8 & $2.9 \pm 1.0$ & $4.5 \pm 1.4$ & 412 \\
\hline 2 & 1 & $\begin{array}{l}\mathrm{A}-\mathrm{LC} \\
(2)\end{array}$ & $\begin{array}{l}\text { I-HC } \\
\text { Y190T } \\
\text { W55T } \\
(3)\end{array}$ & 80 & 6 & $1.8 \pm 0.6$ & $2.3 \pm 0.9$ & 6 \\
\hline 1 & 1 & $\begin{array}{l}\text { A-LC } \\
\text { (1) }\end{array}$ & $\begin{array}{l}\text { I-HC } \\
\text { Y190T } \\
(4)\end{array}$ & 80 & 8 & - & - & 31 \\
\hline
\end{tabular}

Single-channel currents were recorded from cells transfected with A-HC plus I-LC containing double Y190TW55 binding site mutation (line 1), with A-LC plus I-HC containing the double Y190TW55T (line 2) or single Y190T (line 3) binding site mutations. The arrangements corresponding to the analyzed amplitude classes for each transfection condition are shown. In the schemes, the black cross indicates inactive coupling region, the binding site mutation are shown by small black circles, and high- and low-conductance forms are shown as black and gray circle lines, respectively. $\tau_{0}$ and $\tau_{\mathrm{B}}$ correspond to the longest duration component of the open and burst duration histograms, respectively. Results are shown as mean $\pm S D$ of different patches for each amplitude class. $n$ is the number of recordings that were analyzed for each condition.

(recordings from 30 green fluorescent cells for each transfection combination). Also, single-channel currents at $-70 \mathrm{mV}$ membrane potential in the presence of $2 \mathrm{~mm}$ ACh were not detected (26 patches from green fluorescent cells in which Y190T was the binding site mutant; 29 patches from cells in which W55T was the binding site mutant). Thus, for an intact binding site formed by subunits in which only one contains an active coupling region, the consequences of binding are not conveyed from one face of the binding site to the active coupling region of the opposing subunit.

We next asked whether a coupling region in a subunit flanked by inactive binding sites can contribute to open-channel stability. In contrast to the experiment in Figure 6, this experiment required deliberate placement of binding sites relative to coupling regions within the pentamer. Thus, we coexpressed A-HC and I-LC-Y190T/W55T subunits, for which channel openings with amplitude of $8 \mathrm{pA}$ correspond to receptors with three consecutive active binding sites and an inactive coupling region in the subunit that inactivates the remaining two sites (Table 2, Fig. 7). Our previous studies showed that receptors with three consecutive binding sites and five active coupling regions exhibit reduced $\tau_{\mathrm{O}}$ of $5.3 \mathrm{~ms}$ and $\tau_{\mathrm{B}}$ of $9.7 \mathrm{~ms}$ (Rayes et al., 2009). However, in the present experiment, channel openings with amplitude $8 \mathrm{pA}$ show reduced $\tau_{\mathrm{O}}$ of $2.9 \mathrm{~ms}$ and $\tau_{\mathrm{B}}$ of $4.5 \mathrm{~ms}$. Thus, a coupling region in a subunit flanked by two inactive binding sites still contributes to open-channel stability. 
To further examine the relationship between the positioning of binding sites and coupling regions, we coexpressed A-LC and I-HC-Y190T/W55T subunits. Channel openings with amplitude of $6 \mathrm{pA}$ are expected, corresponding to receptors with one active binding site and two active coupling regions in the subunits that form the site. The resulting channel openings exhibit $\tau_{\mathrm{O}}$ of $1.8 \mathrm{~ms}$ and appear mainly as isolated channel openings as indicated by the similar duration of burst and open components (Table 2, Fig. 7). By comparison, our previous study showed that receptors with one active binding site and five active coupling regions exhibited isolated channel openings with $\tau_{\mathrm{O}}$ of $2.2 \mathrm{~ms}$. Thus, if only one binding site is functional, active coupling regions are required in both subunits that form that site.

The experiment in Figure 6 suggests that a receptor with one functional binding site and one functional coupling region is not capable of channel opening. To further test this interpretation, we coexpressed A-LC with I-HC-Y190T subunits in different ratios and looked for channel openings of $8 \mathrm{pA}$ amplitude (Table 2). Channel activity from this subunit combination was extremely rare. From 31 patch recordings from green fluorescent cells, channel openings of $8 \mathrm{pA}$ amplitude were detected in only 9 recordings, totaling 180 opening events with an overall $\tau_{\mathrm{O}}$ of $150 \mu \mathrm{s}$. The frequency of these opening events was so low that the possibility cannot be excluded that they arose from spontaneous channel opening. Thus, in agreement with the experiment in Figure 6, a receptor with one functional binding site and one functional coupling region is essentially incapable of channel opening.

\section{Discussion}

The present work shows that the coupling region of each subunit of a model homomeric Cys-loop receptor contributes equal increments to open-channel lifetime. Observation of equal increments indicates that each coupling region contributes independently and symmetrically to the stability of the open channel. The agonist binding sites, by contrast, contribute to open-channel stability interdependently and asymmetrically. Although the coupling regions contribute independently and symmetrically, their action is allosteric because open-channel stability is altered even when an inactive coupling region is present in a subunit flanked by disabled binding sites. The independence and symmetry of the coupling regions mirror that of the M2 transmembrane domains (Filatov and White, 1995; Labarca et al., 1995), suggesting a one-to-one relationship between these distinct but physically linked functional domains.

Our previous study of the same model homomeric receptor established that agonist occupancy of three of the five identical binding sites was sufficient to maximally stabilize the open channel, as long as one site was formed at a subunit interface separated from the other two (Rayes et al., 2009). In this nonconsecutive arrangement of binding sites, bound agonist would contact all five subunits of the pentamer, potentially transmitting a structural perturbation to all five coupling regions. In this case, the minimum number of binding sites required for maximal re-

\section{Requirements for Maximal Open Channel Lifetime

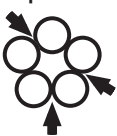 \\ 3 non consecutive binding sites \\ 5 active coupling regions}

Requirements for Detectable Channel Opening

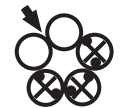

1 binding site

2 active coupling regions

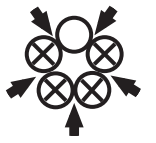

5 binding sites

1 active coupling region

Figure 8. Pentameric arrangements of subunits required for maximal channel lifetime and channel opening. The arrows indicate functional binding sites, and the black crosses indicate inactive coupling regions.

sponse may not be the same as the minimal number of coupling regions. In support of this model, consecutive placement of three binding sites provided less stabilization of the open channel, possibly because agonist contacted only four of the five subunits. However, if bound agonist only affected the coupling region of the subunit that formed one face of the binding site, the minimum number of binding sites required for maximal response would be the same as the minimal number of coupling regions. Together, the present and previous studies support the first possibility, namely that maximal channel lifetime is achieved with three nonconsecutive binding sites and five functional coupling regions (Fig. 8).

We also show that the coupling region can contribute to openchannel stability even when present in a subunit flanked by nonfunctional binding sites. Demonstration of such an allosteric contribution was accomplished by incorporating a subunit with an inactive coupling region that also harbored the mutations Y190T and W55T, which inactivated the principal or complementary faces of the two flanking binding sites. The resulting 
receptor with three consecutive binding sites and four functional coupling regions elicited much briefer channel openings than a receptor with three consecutive binding sites and five functional coupling regions. Thus, a coupling region can contribute to open-channel stability independently of the binding sites.

The final step in the biological response is movement of the M2 transmembrane domains, which connect directly to the coupling region through the M2-M3 linkages. Previous studies of the muscle $\mathrm{AChR}$ showed that the $\mathrm{EC}_{50}$ for activation decreased linearly with an increase in the number of M2 domains harboring a Leu-to-Ser or -Thr substitution at the central 9' position (Filatov and White, 1995; Labarca et al., 1995); the decreases in $\mathrm{EC}_{50}$ arose at least in part through increases in open-channel lifetime. The independent and symmetrical contributions of the M2 domains to open-channel lifetime were suggested to arise through independent and symmetrical movements of the M2s. The analogous independent and symmetrical contributions of the coupling regions suggest a one-to-one relationship between motion of the coupling regions and motion of the M2s. Maximal open-channel lifetime thus requires movement of five coupling regions and five M2 domains.

In addition to addressing requirements for maximal openchannel stability, our study also addresses requirements for opening of the channel regardless of stability. We find that receptors can open even with one functional binding site and two contiguous and functional coupling regions, or with five functional binding sites and one functional coupling region (Fig. 8). These minimal requirements for channel opening suggest that either movement of fewer than five M2 domains is enough to fully open the channel, or that movement of fewer than five M2 domains induces a regenerating movement of the remaining M2s.

Over the past several years, structural and computational studies favor one of these two possibilities. In the apparently closed state of the Torpedo AChR, the narrowest region of the channel is wide enough to accommodate a hydrated sodium ion (Unwin, 2005). However, hydrophobicity of the channel severely limits the ingress of water molecules producing a negligible rate of ion translocation (Beckstein and Sansom, 2006; Corry, 2006; Wang et al., 2008). In the open state, the increased channel diameter increases water occupancy and consequently ion conduction. Although studies of model hydrophobic nanotubes suggest that small changes in channel diameter can produce substantial increases in water occupancy (Hummer et al., 2001; Beckstein and Sansom, 2004), it seems unlikely that movement of only one coupling region and its associated M2 would allow ingress of water sufficient for full ion conduction. Instead, movement of one coupling region and its M2 may promote a regenerating movement of the other four M2s that produces a fully conducting channel. Our study suggests that the M2s associated with an inactive coupling region, although enabling full ion conductance, are unable to stabilize the open channel and thus reduce openchannel lifetime.

\section{References}

Bartos M, Corradi J, Bouzat C (2009) Structural basis of activation of Cysloop receptors: the extracellular-transmembrane interface as a coupling region. Mol Neurobiol 40:236-252.

Beckstein O, Sansom MS (2004) The influence of geometry, surface character, and flexibility on the permeation of ions and water through biological pores. Phys Biol 1:42-52.

Beckstein O, Sansom MS (2006) A hydrophobic gate in an ion channel: the closed state of the nicotinic acetylcholine receptor. Phys Biol 3:147-159.

Bouzat C, Bren N, Sine SM (1994) Structural basis of the different gating kinetics of fetal and adult acetylcholine receptors. Neuron 13:1395-1402.
Bouzat C, Gumilar F, Esandi MC, Sine SM (2002) Subunit-selective contribution to channel gating of the M4 domain of the nicotinic receptor. Biophys J 82:1920-1929.

Bouzat C, Gumilar F, Spitzmaul G, Wang HL, Rayes D, Hansen SB, Taylor P, Sine SM (2004) Coupling of agonist binding to channel gating in an ACh-binding protein linked to an ion channel. Nature 430:896-900.

Bouzat C, Bartos M, Corradi J, Sine SM (2008) The interface between extracellular and transmembrane domains of homomeric Cys-loop receptors governs open-channel lifetime and rate of desensitization. J Neurosci 28:7808-7819.

Chakrapani S, Bailey TD, Auerbach A (2004) Gating dynamics of the acetylcholine receptor extracellular domain. J Gen Physiol 123:341-356.

Corry B (2006) An energy-efficient gating mechanism in the acetylcholine receptor channel suggested by molecular and Brownian dynamics. Biophys J 90:799-810.

Eiselé JL, Bertrand S, Galzi JL, Devillers-Thiéry A, Changeux JP, Bertrand D (1993) Chimaeric nicotinic-serotonergic receptor combines distinct ligand binding and channel specificities. Nature 366:479-483.

Filatov GN, White MM (1995) The role of conserved leucines in the M2 domain of the acetylcholine receptor in channel gating. Mol Pharmacol 48:379-384.

Grutter T, de Carvalho LP, Dufresne V, Taly A, Edelstein SJ, Changeux JP (2005) Molecular tuning of fast gating in pentameric ligand-gated ion channels. Proc Natl Acad Sci U S A 50:18207-18212.

Hamill OP, Marty A, Neher E, Sakmann B, Sigworth FJ (1981) Improved patch-clamp techniques for high-resolution current recording from cells and cell-free membrane patches. Pflugers Arch 391:85-100.

Hummer G, Rasaiah JC, Noworyta JP (2001) Water conduction through the hydrophobic channel of a carbon nanotube. Nature 414:188-190.

Kash TL, Jenkins A, Kelley JC, Trudell JR, Harrison NL (2003) Coupling of agonist binding to channel gating in the $\mathrm{GABA}_{\mathrm{A}}$ receptor. Nature 421:272-275.

Kelley SP, Dunlop JI, Kirkness EF, Lambert JJ, Peters JA (2003) A cytoplasmic region determines single-channel conductance in $5-\mathrm{HT}_{3}$ receptors. Nature 424:321-324.

Labarca C, Nowak MW, Zhang H, Tang L, Deshpande P, Lester HA (1995) Channel gating governed symmetrically by conserved leucine residues in the M2 domain of nicotinic receptors. Nature 376:514-516.

Lee WY, Sine SM (2005) Principal pathway coupling agonist binding to channel gating in nicotinic receptors. Nature 438:243-247.

Lee WY, Free CR, Sine SM (2009) Binding to gating transduction in nicotinic receptors: Cys-loop energetically couples to pre-M1 and M2-M3 regions. J Neurosci 29:3189-3199.

Liu Y, Dilger JP (1991) Opening rate of acetylcholine receptor channels. Biophys J 60:424-432.

Mercado J, Czajkowski C (2006) Charged residues in the $\alpha 1$ and $\beta 2$ pre-M1 regions involved in $\mathrm{GABA}_{\mathrm{A}}$ receptor activation. J Neurosci 26:2031-2040.

Rayes D, Spitzmaul G, Sine SM, Bouzat C (2005) Single channel kinetic analysis of chimeric $\alpha 7-5 \mathrm{HT}_{3 \mathrm{~A}}$ receptors. Mol Pharmacol 68:1475-1483.

Rayes D, De Rosa MJ, Sine SM, Bouzat C (2009) Number and locations of agonist binding sites required to activate homomeric Cys-loop receptors. J Neurosci 29:6022-6032.

Sine SM (2002) The nicotinic receptor ligand binding domain. J Neurobiol 53:431-446.

Sine SM, Engel AG (2006) Recent advances in Cys-loop receptor structure and function. Nature 440:448-455.

Sine SM, Kreienkamp HJ, Bren N, Maeda R, Taylor P (1995) Molecular dissection of subunit interfaces in the acetylcholine receptor: identification of determinants of alpha-conotoxin M1 selectivity. Neuron 15:205-211.

Thompson AJ, Lester HA, Lummis SC (2010) The structural basis of function in Cys-loop receptors. Q Rev Biophys 43:449-499.

Unwin N (2005) Refined structure of the nicotinic acetylcholine receptor at $4 \AA$ A resolution. J Mol Biol 346:967-989.

Wang HL, Cheng X, Taylor P, McCammon JA, Sine SM (2008) Control of cation permeation through the nicotinic receptor. PLoS Comput Biol 4:e41.

Xiu X, Hanek AP, Wang J, Lester HA, Dougherty DA (2005) A unified view of the role of electrostatic interactions in modulating the gating of Cys loop receptors. J Biol Chem 280:41655-41666. 